\title{
PANCREATIS ASSOCIATED WITH COVID19 AND RELATIONSHIP WITH A HEALTHY LIFESTYLE
}

\author{
Gabriel Miranda* ., Maria Preciado' \& Luis Gallo'
}

Nava Military Medical Specialist in neurology, neurophysiology and sleep diseases, Mexico

Lamar Guadalajara University.

\begin{abstract}
In December of 2019 a severe acute respiratory syndrome emerged in Wuhan and rapidly spread through China subsequently was declared a pandemic on march 2020. Transmission of this virus was linked to direct exposure to Huanan Seafood Wholesale Market of Wuhun makin. Common COVID 19 symptoms are cough (50\%), fever (43\%), myalgia (36\%), headache (34\%), dyspnea (29\%), sore throat (20\%), Diarrhea (19\%), Nausea and vomiting (12\%), anosmia and agnosia (10\%) (7) and abdominal pain being one of the most common symptoms. However, increase data has been reported associating COVID 19 and acute pancreatitis.During this clinical research we will analyze potential risk for having a pancreatitis and how we can relate this to healthy patterns in a patient, considering age, lifestyle, diet, and doing exercise daily we want to see if there is a relationship as to why most patients during this study were mostly female, overweight and if there is a potential common reason such as obesity, alcoholism, smoking, gallstones and hypertriglyceridemia.
\end{abstract}

\section{Keywords: Nil}




\section{INTRODUCTION}

In December of 2019 a severe acute respiratory syndrome emerged in Wuhan and rapidly spread through China subsequently declared a pandemic on march 2020. This virus belongs to an RNA enveloped group of viruses of ssRNA that had previously been reported in bats in china. (1) This virus belongs to the corona virus family which comprise an alpha, beta, delta and gamma coronavirus, with an spherical external spike protein and displays a characteristic crown shape when observed under a microscope giving it its name. There are six know coronaviruses that typically cause infection in humans although generally cause mild cold like symptoms.

Following the outbreaks of unexplained pneumonia in Wuhan the new coronavirus was identified naming it COVID 19. (2)

Transmission of this virus was linked to direct exposure to Huanan Seafood Wholesale Market of Wuhun making, the first mechanism associated was animal human transmission, however subsequent cases where not associated with this mechanism, therefore human to human transmission was proposed and further acknowledging that that asymptomatic patients are the most dangerous for transmission. (3) Symptomatic patients commonly present with cough (50\%), fever (43\%), myalgia (36\%), headache (34\%), dyspnea (29\%), sore throat (20\%), Diarrhea (19\%), nausea and vomiting (12\%), anosmia and agnosia (10\%) (4) and abdominal pain being one of the most common symptoms. (5) Nonetheless, through all the year cases of COVID 19 and pancreatitis have been reported.

Pancreatitis has been commonly associated with gallstones and alcohol. Furthermore, causes of pancreatitis are no limited to this and rather extend to: autoimmune, hypertriglyceridemia, post endoscopic retrograde cholangiopancreatography, genetic risk, pancreatic duct injury and medications (6) such as azathioprine, 6-mercaptopurine, didanosine, valproic acid, ACE inhibitors, mesalamine, metronidazole, omeprazole, macrolides. (7) Nevertheless, about $10 \%$ of cases had been associated with infections such as viruses, bacteria and parasites in which Coxsackie virus, cytomegalovirus (CMV), human immunodeficiency virus (HIV), herpes simplex virus (HSV), mumps, varicella-zoster virus, mycoplasma, legionella, salmonella, leptospira, toxoplasmosis, cryptosporidium, and ascaris are the most common etiologies. (8) We present an analysis of 44 cases associated with pancreatitis and COVID 19 reported in 24 articles.

\section{METHODS}

This is a prospective study enrolling 44 patients that have presented pancreatitis associated to covid 19, all cases were studied case by case, with patients of different ages and gender enrolled on it, each case with different treatment, signs, symptoms and individuality, all of this patients took a SARS-COV2 test and PCR to confirm their diagnosis. 
Clinical signs, aswell as radiologic signs were taken into account to diagnose pancreatitis in the patients that are presented in the clinical study, the study was performed for 24 patients

\section{DISCUSSION}

During this study we recollected data for 44 patients from 24 different studies in which most patients had presented similar symptoms, most of the patients were over 50 years old, we believe that having a healthy lifestyle can decrease the possibility of having a pancreatitis associated with covid 19, we have that at least $25 \%$ of the patients in our study had Hypertension or Diabetes Mellitus, we know that maintaining a weight where an individual has a waist circumference inferior to 90 will decrease by far the possibility that they get on further years in their life a chronic disease it will even decrease the possibility of having pancreatic cancer or developing gallstones associated to being overweight or obese.

Exercising is important we know that in United States the major reason of death and mortality are hearth related diseases which are also associated to obesity and overweight, nowadays the two countries with the biggest overweight population are both Mexico and United States they are the countries with the most overweight children in the World.

We will relate covid 19 to pancreatitis but what are the major reasons for this happening, it most probably is related to being overweight and having obesity, most of the patients that have died in the whole world due to covid 19 had chronic diseases related to them such as Hypertension which we know is mostly related to triglycerides and inflammatory debris alongside the arterial walls which eventually will lead you to hypertension or Diabetes Mellitus which is related to end glycosylated products also grouping alongside the arterial walls, it is important to note that also heart related diseases such as even having a myocardial infarction is mostly related to inflammatory debris and hypertriglyceridemia that have accumulated alongside the coronary arteries such as the circumflex artery, the left descending artery and the right coronary artery.

We recollected data from 24 articles that were screened and found eligible for data extraction. These articles reported cases of COVID 19 and pancreatitis, these patients seek for clinical attention for treatment of fever (36\%), nausea and vomiting (31\%), diarrhea (25\%), abdominal pain (20\%), severe presentation/pneumonia (20\%), cough (13\%), all of them were diagnosed with COVID 19 at the admission to the hospital. From these group of patients, the incidence of females was observed to be slightly bigger than with males, the observed ratio was 23:21 patients. (Table 1) 


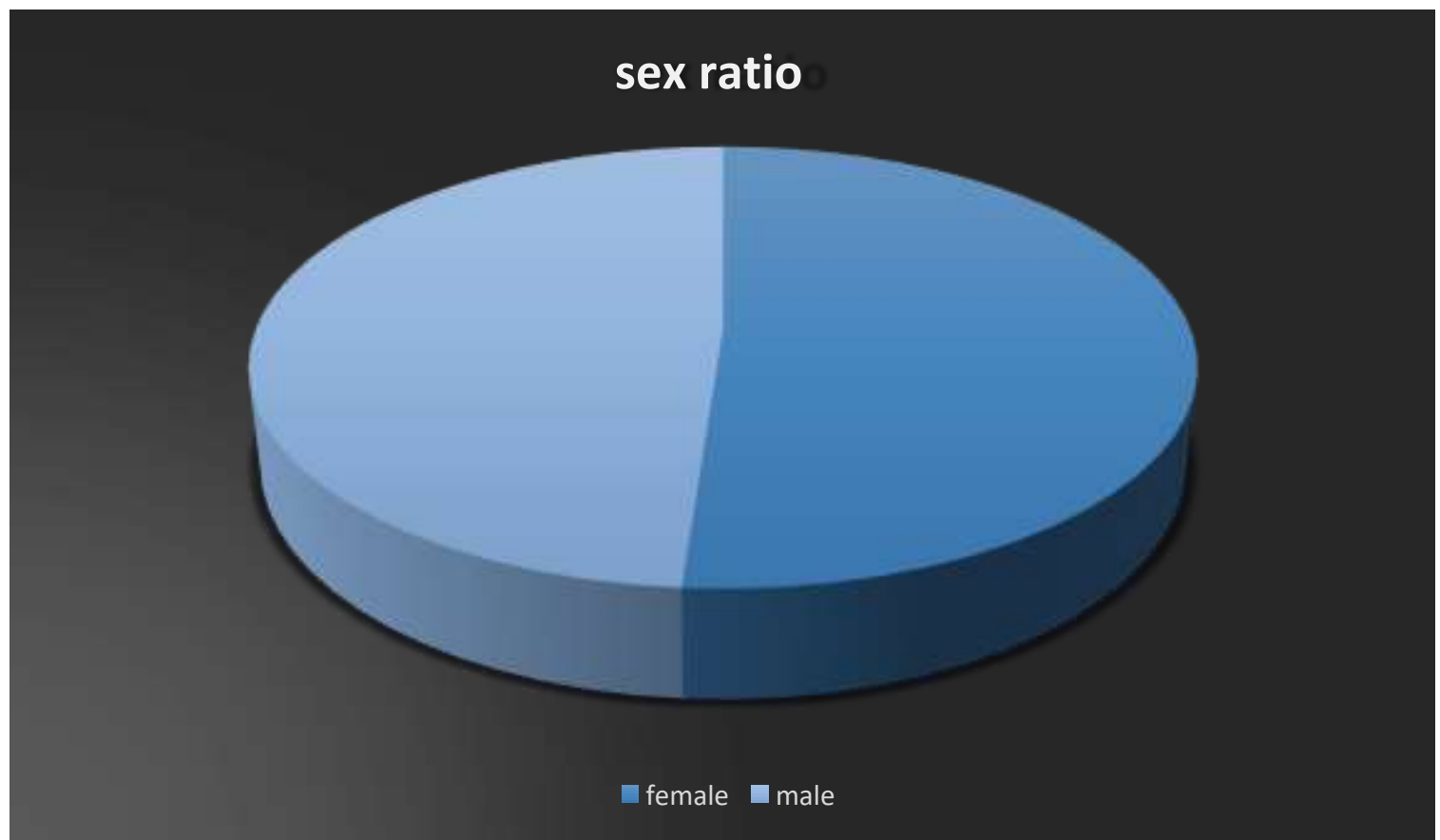

The mean age of the group of patients was 50 , with a mode of 36 and median of 55 years old. Our data reported that the youngest patient had 24 years old and was a male and our oldest patient was a woman of 90 years old. Table 2 shows the comparison of age between females and males. Reporting 6 males had an age between 21-40 years old, 7 males had between $41-60$ years and lastly 8 patients had ages between 81-100, from females the data reported 8 females between 21 40 years old, 6 felled in 41-60 years old, 7 had between 61-80 years and lastly 1 woman felled in the category of 81-100 years old. (Table 2)

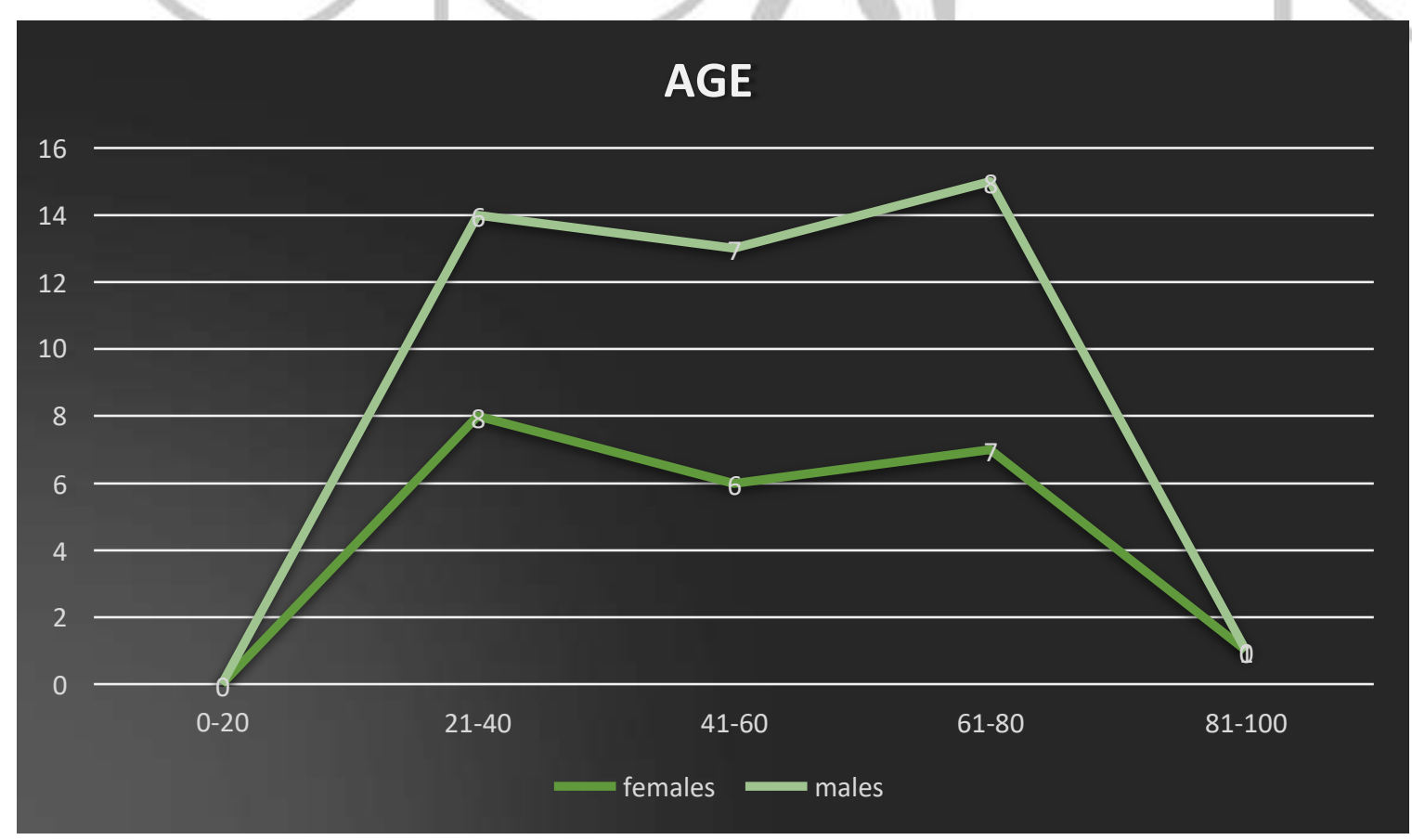


On tables 3 and 4 we demostrate 18 of the patients denied any comorbidities while 26 reported at least 1 comorbid from the following: hypertension $28 \%$, diabetes mellitus $24 \%$, obesity $8 \%$, asthma $8 \%$, smoking $4 \%$ and $8 \%$ reported other diseases.

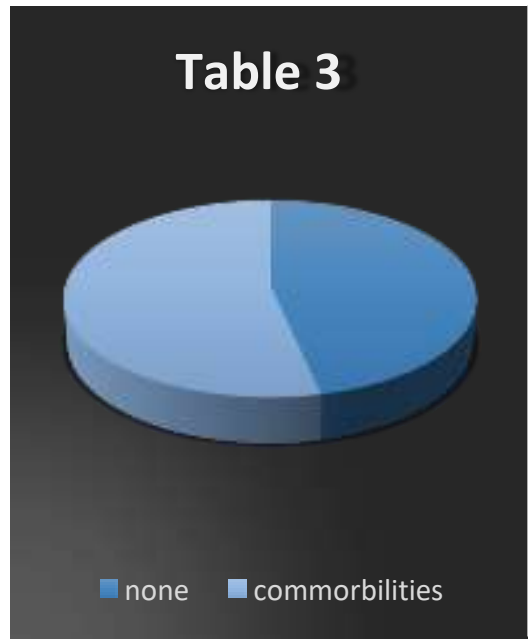

\section{Table 4}
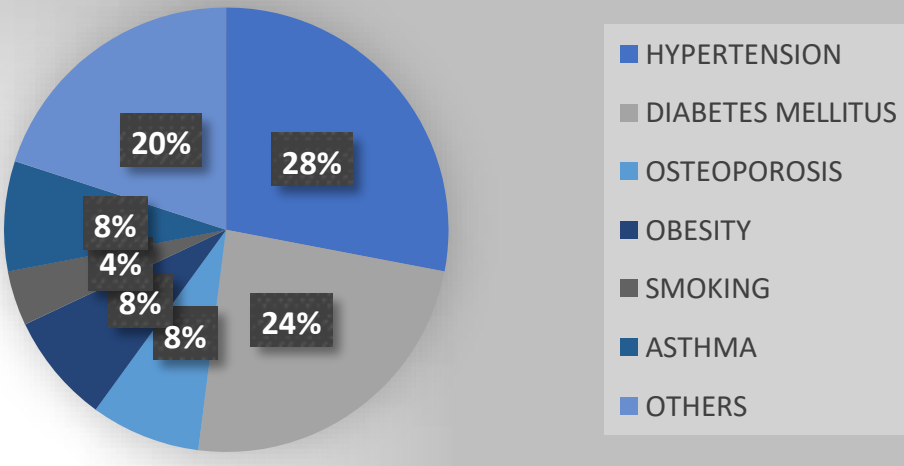

We also observed the drugs used in these patients in view of the fact that some acute pancreatitis have been associated to certain drugs.

\section{DRUGS}

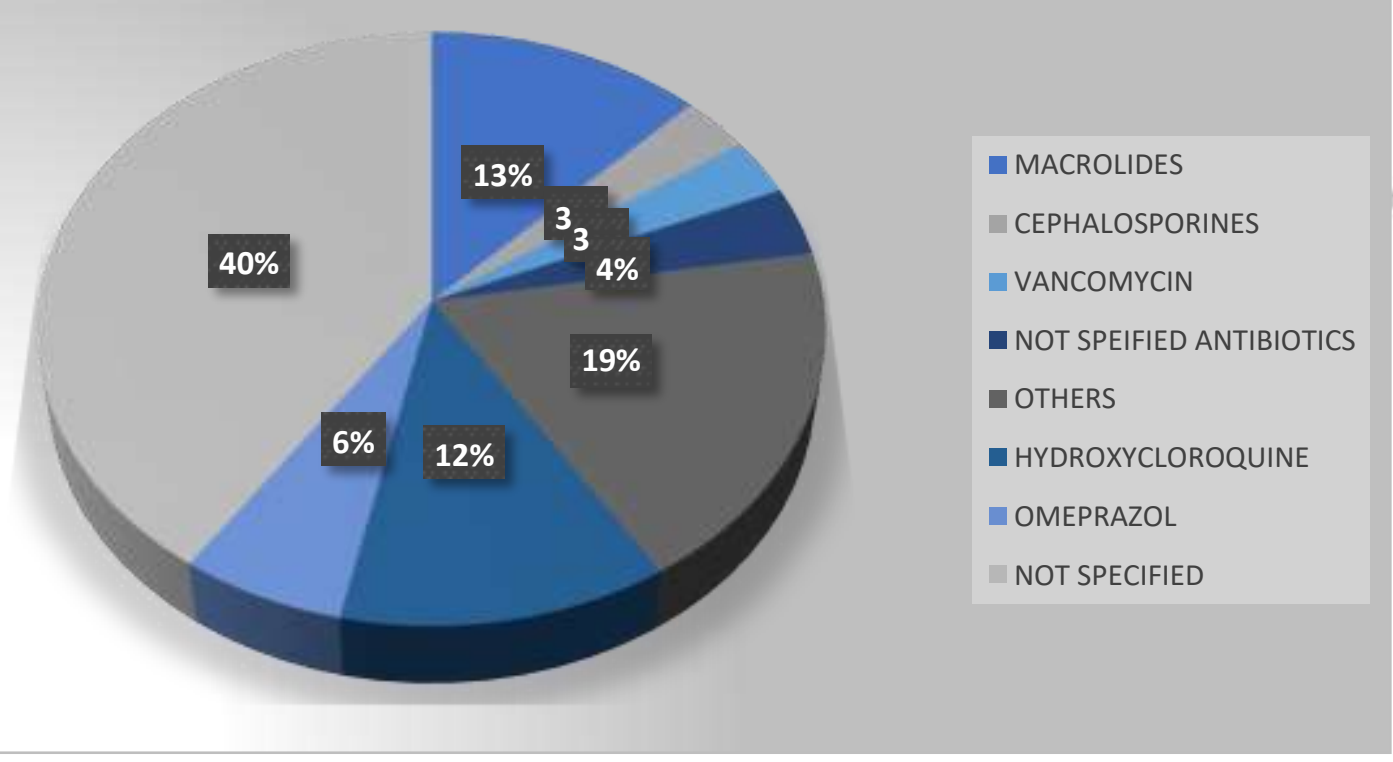




\section{CONCLUSIONS}

We see that most patients presented general symptoms nausea, vomiting, fever and abdominal pain, which are characteritistic of pancreatitis we can also add that most patients were female which happens to be that most women over 40 tend to have overweight or obesity, being that most of the patients that we have in our sample is over 50 years old, we believe that although this is more an informative research that the importance of a healthy lifestyle, such as a diet, exercising, having habits towards a positive lifestyle can prevent in an enormous amount the impact of having covid, many of the patients in this study were female, it is important to note that women tend to have obesity and overweight on later years due to the fact of pregnancy, the fact that women over 50 and with menopause tend to keep more lipids on their body and increase weight as they age, aswell as the fact that smoking is more prevalent in women than in men which also happens to be alongside obesity one the two major causes for pancreatic cancer more specifically the biggest cause for pancreatic cancer is smoking, also is important to note that women tend to have gallbladder and biliary tree obstructions alongside choledocolitiasis and cholangitis is a lot more prevalent In women which can put in risk a person towards pancreatic problems more than a male individual.

\section{BIBLIOGRAPHY.}

Abdul Mannan Baig,Evidence of COVID-19 virus targeting the CNS: Tissue distribution, host- virus interaction, and proposed neutropic mechanisms, ABCS Chemical neuroscience 2020

2- Jeong-Min Kim, Identification of coronavirus isolated from a patient in korea with COVID 19, Public Health and Research Perspectives 2020

3-

Marco Cascella, Features,

Evaluation, and Treatment of coronavirus, STATPearls NCBI, 2020

4 Knneth Mclntosh, Coronavirus disease 2019 (COVID 19 ) clinical features, UPTODATE 2020

5 Santhi Swaroop Vege, Etiology of acute Pancreatitis, UPTODATE 2020 6Nabeeha Mohy- Ud-Din, Pancreatitis, STATPearls NCBI 2020

7- Nison Badalov, Drug induced acute pancreatitis: an evidence- based review,Clinical Gastroenterology and Hematology 2020 